\title{
COMPARATIVE ANALYSIS OF FACE RECOGNITION BASED ON SIGNIFICANT PRINCIPAL COMPONENTS OF PCA TECHNIQUE
}

\author{
Manzoor Ahmad Lone \\ Department of Computer Sciences and Engineering, \\ University of Kashmir, North Campus, J \& K, India
}

\begin{abstract}
Face recognition systems have been emerging as acceptable approaches for human authorization. Face recognition help in searching and classifying a face database and at a higher level help in identification of possible threats to security. In face recognition problem, the objective is to search a face in the reference face database that matches a given subject. The task of face recognition involves the extraction of feature vectors of the human face from the face image for differentiating it from other persons [6]. In this work, the comparative analysis is done based on the varying number of highly significant principal components (Eigenvectors) of PCA for face recognition. Experimental results show a small number of principal components of PCA are required for matching. PCA technique is a statistical technique, it reduces the dimension of the search space that best describes the images.
\end{abstract}

Keywords: Covariance Matrix, Eigenvector, Eigenvalue, Euclidean Distance, ORL, PCA.

Cite this Article: Manzoor Ahmad Lone, Comparative Analysis of Face Recognition Based on Significant Principal Components of PCA Technique, International Journal of Computer Engineering and Technology, 10(1), 2019, pp. 94-101.

http://iaeme.com/Home/issue/IJCET?Volume=10\&Issue $=1$

\section{INTRODUCTION}

Principal Component Analysis is a technique, which uses sophisticated underlying mathematical principles to transform a number of correlated variables into a smaller number of variables called principal components $[3,8]$. PCA reduces the large dimensionality of the data space (observed variables) to a smaller intrinsic dimensionality of feature space (independent variables), which are needed to describe the data efficiently. PCA can do prediction, data redundancy removal, feature extraction, data compression, etc. The Principal component analysis is also known as eigenface method, in this method the images are 
projected onto the facial value so called eigenspace [2,7]. The eigenvectors are ordered on the basis of eigenvalues, each one accounting for a different amount of the variation among the face images. Each image location contributes more or less to each eigenvector. PCA approach reduces the dimension of the data by means of data compression basics [1] and reveals the most effective low dimensional structure of facial patterns. This reduction in dimension removes information that is not useful [5] and precisely decomposes the face structure into uncorrelated components known as eigenfaces. This method is preferred because of its simplicity, speed and learning capability [4]. PCA is a useful statistical technique that has found application in fields such as face recognition and image compression and is a common technique for finding patterns in data of high dimension.

\section{ORL FACE DATABASE}

ORL face database consist of a set of faces taken at the Olivetti Research Laboratory in Cambridge, United Kingdom [10]. ORL face database has been used to benchmark in many face identifying systems. It contains a total of 400 face images of forty different persons, ten images per person. For each subject, it contains 10 face images under different facial expressions, lighting conditions and poses. All the face images are taken against a dark homogeneous background with the persons in an up-right, frontal position, with tolerance for some tilting and rotation of up to about 20 degrees. The images have a resolution of $92 \times 112$ pixels, grayscale and in bitmap file format (.bmp). There is diversity in the images of different subjects like a subject has beard, glasses, moustaches etc.

\subsection{Face recognition using Principal Component Analysis (PCA)}

Figure. 1 shows the flowchart of a PCA based face recognition system [9]. Preliminarily some preprocessing task is done such as resizing the image size. ORL face database images size is $92 \times 112$ and after resizing the face image size is $92 \times 92$. For implementing face recognition system based on the PCA technique [3,8], we follow the three steps: Face preprocessing, feature vector extraction and recognition step.

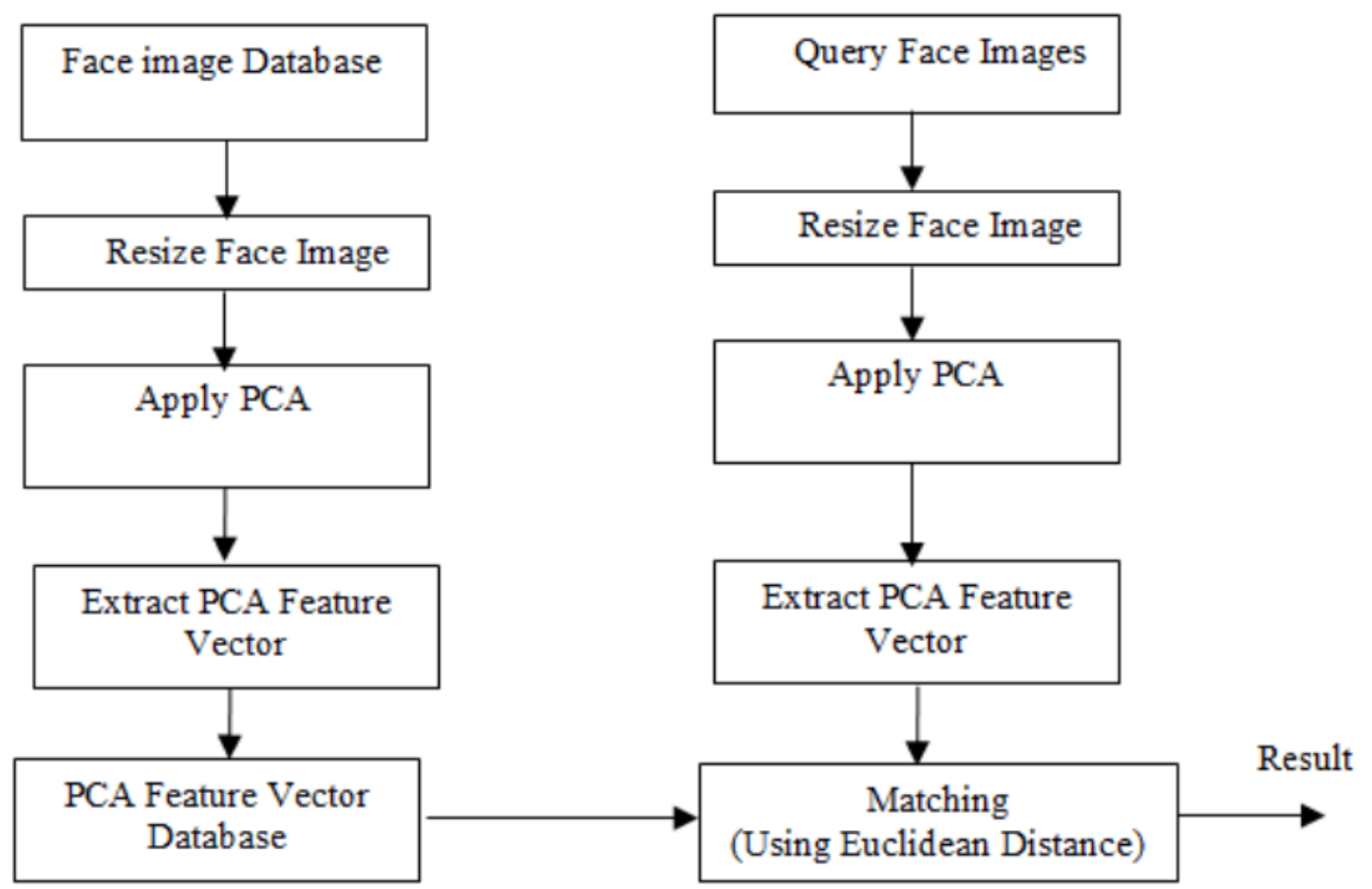

Figure 1 Flow chart of face recognition system based on PCA 
Comparative Analysis of Face Recognition Based on Significant Principal Components of PCA Technique

\subsection{Steps}

1. A set of $M$ training images (I1, I2, I3,.., IM) with size $N \times M$ are changed into $N \times N$ and are then represented by a column vector of size $1 \times \mathrm{N} 2$.

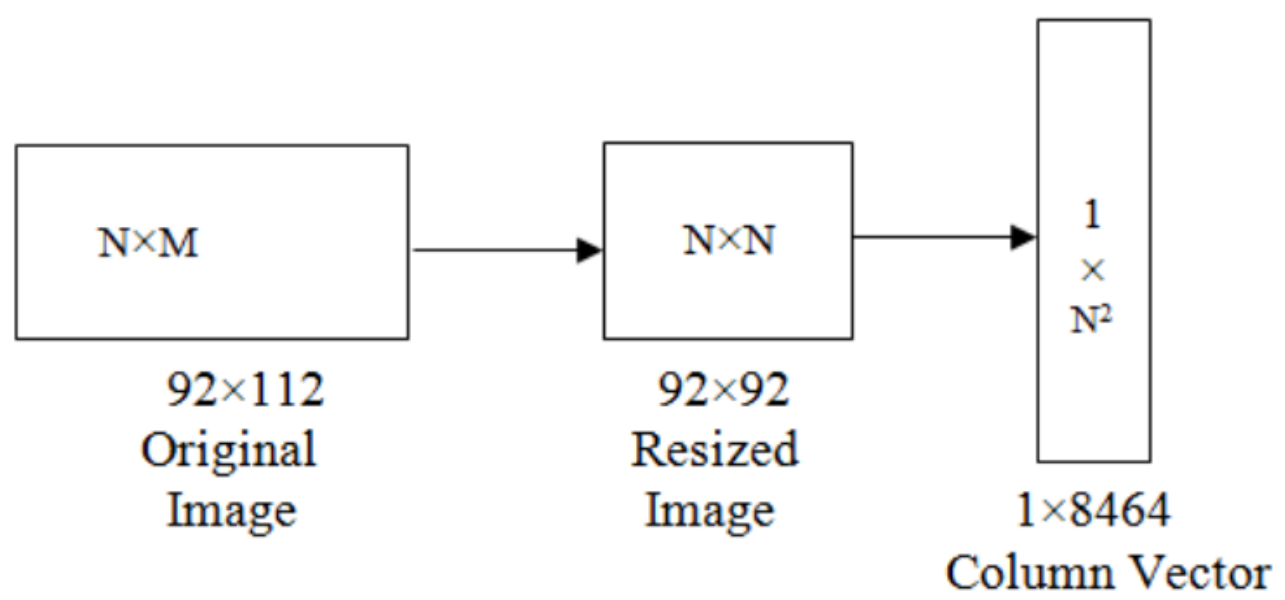

2. The average ( $\Psi)$ of the training set image is calculated and is defined by the following mathematical formula:

$$
\Psi=\frac{\sum_{n=1}^{M} T_{n}}{M}
$$

3. The average face vector $\Psi$ is subtracted from each trainee image I and stored in $\Phi$.

$$
\Phi=\mathrm{I}_{\mathrm{i}}-\Psi \text { (Deviation From mean vector) }
$$

4. From $\Phi$, the Covariance Matrix " $C$ " is calculated as follows:

$$
\mathrm{C}=\mathrm{AA}^{\mathrm{T}}
$$

Where, $\mathrm{A}=\left[\Phi_{1}, \Phi_{2}, \Phi_{3}, \ldots, \Phi_{\mathrm{M}}\right]$

5. Calculate the eigenvalues $\lambda i$ and eigenvectors ui of the covariance matrix $C$ with $\mathrm{N} 2 \mathrm{xN} 2$ dimension and it is impractical to make such number of calculations. To reduce the number of calculations, the following steps are followed:

$$
\text { Let } \quad \mathrm{L}=\mathrm{A}^{\mathrm{T}} \mathrm{A}
$$

Calculate $v_{i}$ of equation-(e)

$$
\begin{gathered}
\mathrm{A}^{\mathrm{T}} \mathrm{Avi}=\mathrm{u}_{\mathrm{i}} \mathrm{v}_{\mathrm{i}} \\
\mathrm{AA}^{\mathrm{T}} \mathrm{Av}_{\mathrm{i}}=\mathrm{u}_{\mathrm{i}} \mathrm{A} v_{\mathrm{i}} \\
\mathrm{CAv}_{\mathrm{i}}=\mathrm{u}_{\mathrm{i}} \mathrm{A} v_{\mathrm{i}} \quad \text { from }(\mathrm{c}) \\
\mathrm{C} u_{i}=u \mathrm{~A} v_{i}, \quad\left\{\text { where } u_{i}=\mathrm{A} v_{i}\right\}
\end{gathered}
$$

Hence $\mathrm{C}$ and $\mathrm{L}$ have the same eigenvalues and their eigenvectors are related by $u_{i}=\mathrm{A} v_{i}$, Where $v_{i}$ is an eigenvector of $\mathrm{L}=\mathrm{A}^{\mathrm{T}} \mathrm{A}$. From this simple proof, we can see that $\mathrm{A} v_{i}$ is an 
eigenvector of $C=A A^{T}$. The $M$ eigenvectors of $L=A^{T} A$ are used to find the $M$ eigenvectors of $\mathrm{C}$ that form our eigenface basis:

$$
u_{i}=A v_{i}
$$

Where $u_{i}$, are the eigenvectors i.e. eigenfaces.

6. The eigenvectors with high eigenvalues are retained to classify an image with the help of following formula:

$$
\omega_{\mathrm{i}}=u_{i}^{\mathrm{T}}\left(\mathrm{I}_{\mathrm{i}}-\Psi\right)
$$

Where $u_{i}$ is the $\mathrm{i}^{\text {th }}$ eigenfaces and $\mathrm{i}=1,2,3, \ldots, \mathrm{k}$. The weights are obtained as above to form a vector as follows that describes the contribution of each eigenface in representing the input face image.

$$
\Omega_{\mathrm{i}}^{\mathrm{T}}=\left\{\omega_{1}, \omega_{2}, \omega_{3}, \ldots, \omega_{\mathrm{K}}\right\}
$$

7. Apply the same set of steps to the query image to find:

$$
\omega_{\text {query }}=u_{i}^{\mathrm{T}}\left(\mathrm{I}_{\text {query }}-\Psi\right)
$$

Where $u_{i}$ is the $\mathrm{i}^{\text {th }}$ eigenfaces and $\mathrm{i}=1,2,3, \ldots, \mathrm{k}$.

$$
\Omega^{\mathrm{T}}{ }_{\text {query }}=\left\{\omega_{1}, \omega_{2}, \omega_{3}, \ldots, \omega_{\mathrm{K}}\right\}
$$

8. To determine the match, find the Euclidean distance between the query image feature vector and database image feature vectors as follows:

$$
\mathrm{E}_{\mathrm{d}}=\operatorname{sqrt}\left(\left|\Omega^{\mathrm{T}}{ }_{\text {query }}-\Omega^{\mathrm{T}}{ }_{\mathrm{i}}\right|^{2}\right)
$$

9. First 10 images (as each subject has 10 poses) are displayed with minimum Euclidean distance as shown in snapshot figures. 
Comparative Analysis of Face Recognition Based on Significant Principal Components of PCA Technique

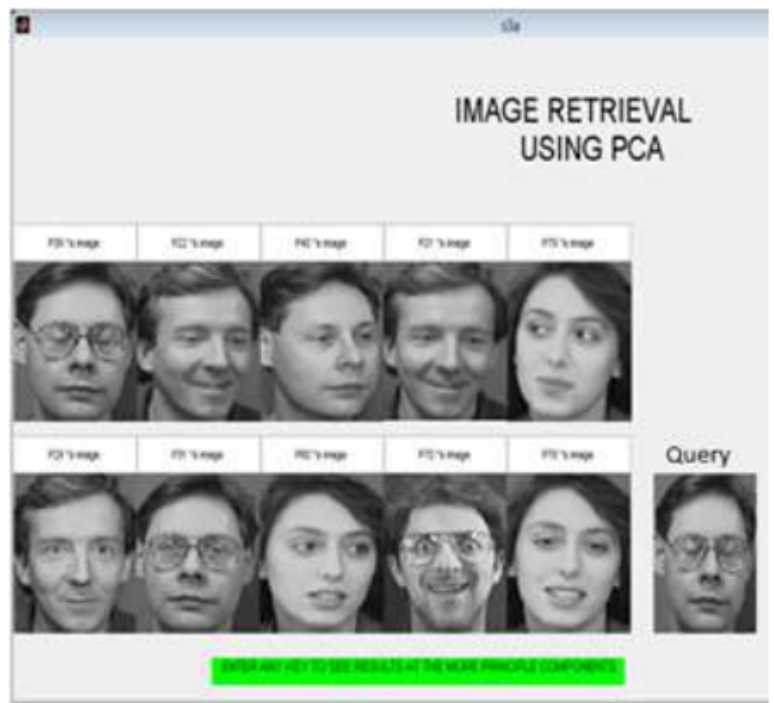

a

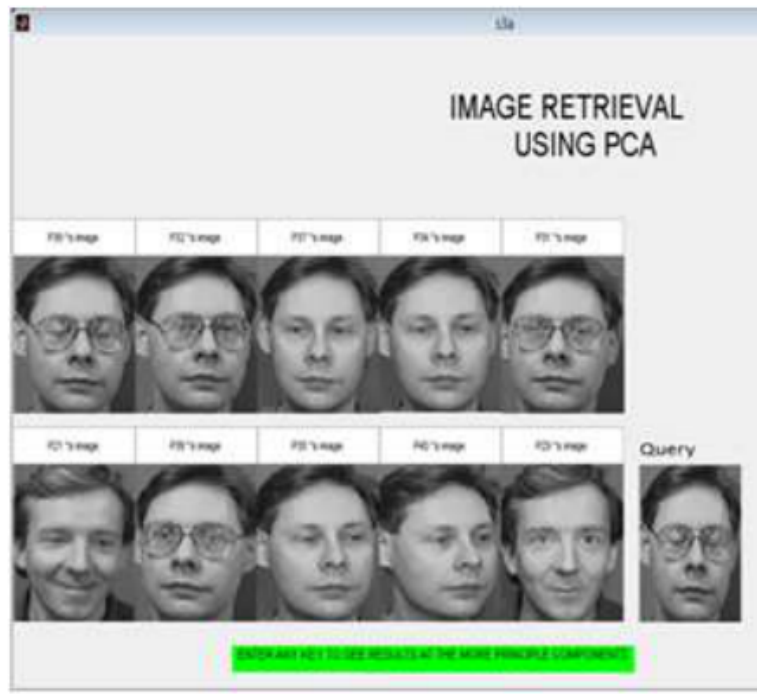

$\mathrm{c}$

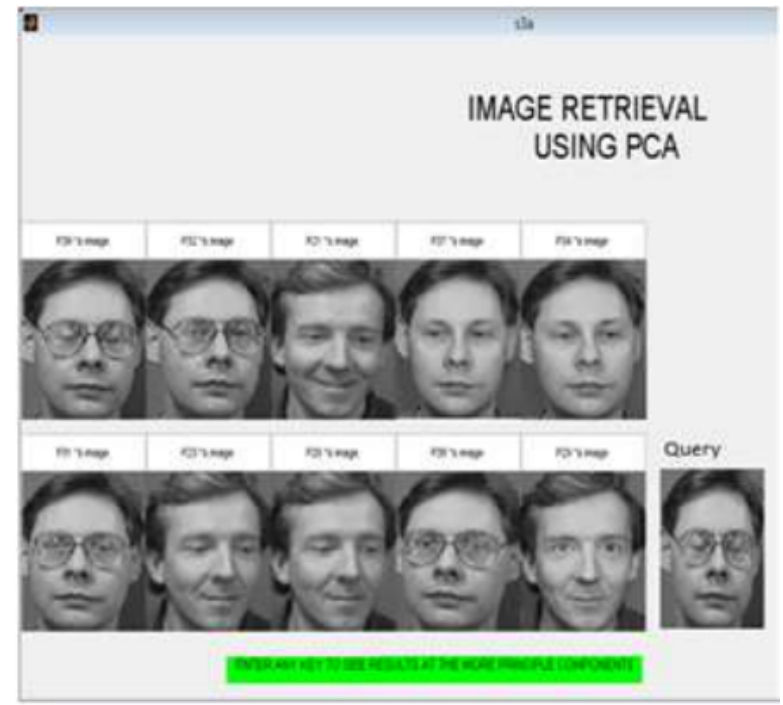

b

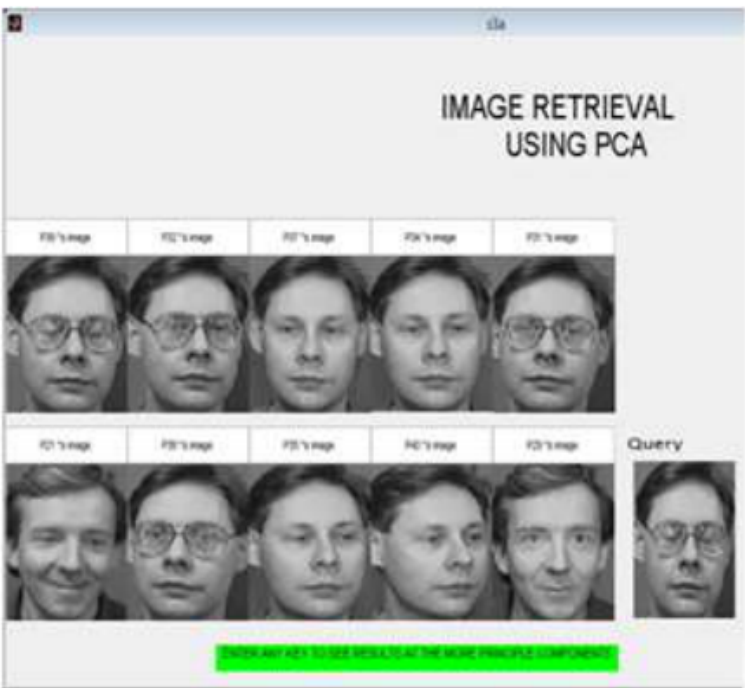

d

Figure a, b, c, $\mathbf{d}$ Recognition/retrieval for one particular pose of a person using a varying number of principal components of PCA in MatLab.

\section{RESULTS AND DISCUSSION}

In this work of face recognition using PCA, the experimental results are obtained in MatLab on Olivetti Research Laboratory (ORL) face image database [7]. ORL face database acts as the reference face database and the experimental results are shown on 100 face images of 10 different persons with 10 poses of each person. The results are shown for various eigenvectors (principal components) with high eigenvalues to determine the match. The results show that from a huge number of eigenvectors (principal components) a small number of significant eigenvectors are required to determine a match between the query image and the database images. 
Table 1 Face Recognition rate of PCA with varying number of Principal Components

\begin{tabular}{|c|c|}
\hline $\begin{array}{l}\text { No. of Principal } \\
\text { Components }\end{array}$ & Recognition Rate (\%) \\
\hline 1 & 46.3 \\
\hline 2 & 71.6 \\
\hline 3 & 76.3 \\
\hline 4 & 84.7 \\
\hline 5 & 84.9 \\
\hline 6 & 85.2 \\
\hline 7 & 86.8 \\
\hline 8 & 87.1 \\
\hline 9 & 87.1 \\
\hline 10 & 87.1 \\
\hline 11 & 87.0 \\
\hline 12 & 87.1 \\
\hline 13 & 87.2 \\
\hline 14 & 87.3 \\
\hline 15 & 87.4 \\
\hline 16 & 87.4 \\
\hline 17 & 87.3 \\
\hline 18 & 87.3 \\
\hline 19 & 87.3 \\
\hline 20 & 87.3 \\
\hline 21 & 87.2 \\
\hline 22 & 87.2 \\
\hline 23 & 87.2 \\
\hline 24 & 87.2 \\
\hline 25 & 87.2 \\
\hline 26 & 87.2 \\
\hline 27 & 87.2 \\
\hline 28 & 87.2 \\
\hline 29 & 87.2 \\
\hline 30 & 87.2 \\
\hline
\end{tabular}


Comparative Analysis of Face Recognition Based on Significant Principal Components of PCA Technique

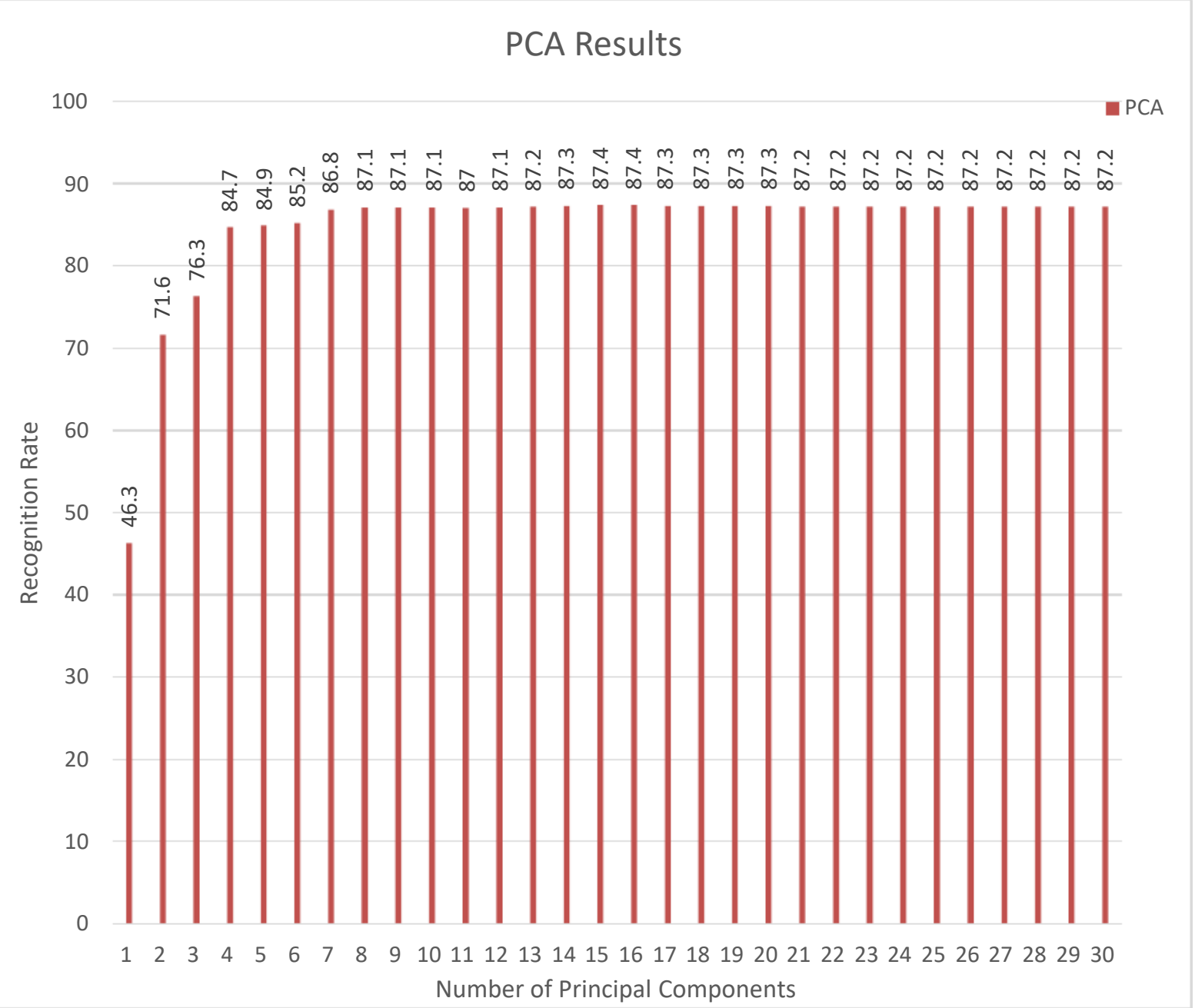

Figure 2 Graphical view of results obtained using PCA at different No. of Principal Components.

\section{CONCLUSION}

In this work, the face recognition system using PCA (Principal Component Analysis) method is implemented in MatLab. The recognition rate is determined on a varying number of principal components and experimental results show that a few significant numbers of principal components are required to determine more than $85 \%$ of the total recognition rate. This clearly indicates that we only require a small number of eigenvectors to determine the similarity between a query image and database images.

\subsection{Future Scope}

This work can be extended to work on a huge set of database images, real time imaging and videos. Moreover, the results of this work can be compared with other different face recognition techniques. In addition to this technique can be fused with artificial neural networks techniques to improve the overall recognition rate. 


\section{REFERENCES}

[1] D. Blackburn, M. Bone, and P. Phillips, "Facial Recognition Vendor Test 2000 Evaluation Report", National Institute of Science and Technology, Gaithersburg, USA, 2000.

[2] T.J. Chin and D. Suter, "A Study of the Eigenface Approach for Face Recognition", Technical Report of Monash University, Dept. Elect. \& Comp. Sys Eng (MECSE 2004) Australia, 1-18, 2004.

[3] G.H. Dunteman, "Principal Component Analysis", Sage publications, 1989.

[4] R. Gross, J. Shi and J. Cohn, "Quo Vadis Face Recognition: Third Workshop on Empirical Evaluation Methods in Computer Vision", Carnegie Mellon University, Pittsburgh, USA, December, 2001.

[5] P.J. Phillips, H. Moon, S. Rizvi and P. Rauss, "FERET Evaluation Methodology for Face Recognition Algorithms", IEEE transaction on pattern analysis and machine intelligence (PAMI 2000), Los Alamitos, USA, 1090-1103, 2000.

[6] D.L. Sirivich and M. Kirby, "Low-Dimensional Procedure for the Characterization of Human Faces", Journal of the Optical Society of America A: Optics, Image Science, and Vision, America, 519-524, 1987.

[7] M. Turk and A. Pentland, "Eigenfaces for Recognition", Journal of Cognitive Neuroscience, 71-86, 1991.

[8] M.A. Turk and A. Pentland, "Face Recognition using Eigenfaces", IEEE Conf. on Computer Vision and Pattern Recognition, 586-591, 1991.

[9] S.M. Zakariya, R. Ali, M.A. Lone, “Automatic Face Recognition Using Multi-Algorithmic Approaches". In: Aluru S. et al. (eds) Contemporary Computing. IC3 2011, Communications in Computer and Information Science, vol 168. Springer, Berlin, Heidelberg, 2011.

[10] The Database of Faces, http://www.cl.cam.ac.uk./research/dtg/attarchive/, Face database.html. 